\title{
BETTI NUMBERS AND THE INTEGRAL CLOSURE OF IDEALS
}

\author{
SANGKI CHOI
}

\section{Introduction.}

Let $(R, \underline{n})$ be a commutative noetherian local ring and $M$ be a finitely generated $R$-module. Then the $i$ th Betti number $b_{i}^{R}(M)$ is the integer $\operatorname{dim}_{R / \underline{n}} \operatorname{Tor}_{i}^{R}(M, R / \underline{n})$. In $1984 \mathrm{~L}$. Avramov asked whether the sequence $b_{i}^{R}(M)$ of Betti numbers is eventually nondecreasing for any finitely generated module $M$ over the local ring $R[1,5.8]$. The answer is known to be true if $(R, \underline{n})$ is local with $\underline{n}^{3}=0[9$, Theorem B, Proposition 3.9] or if $(R, \underline{n})$ is a local Golod ring [10, Corollary 6.5]. Here we give a positive answer to the problem if $(R, \underline{n})$ is a Cohen-Macaulay local ring of multiplicity at most 7 except possibly a complete intersection of multiplicity 6 or 7 .

In section 1 we study the sequence $b_{i}^{R}(M)$ of Betti numbers of $M$ over the local rings of the form $R=S / I,(S, \underline{m})$ a local ring, $I$ an ideal of $S$. Rings of the form $R=S / \underline{m} J, J$ an ideal of $S$, have been studied by Ramras [12], Gover and Ramras [7] and Lescot [9]. In Theorem 1.1 we prove that if $R=S / I$ and the integral closure $\bar{I}$ of the ideal $I$ is properly contained in the integral closure $\bar{J}$ of $J=(I$ is $\underline{m})$, then the sequence $b_{i}^{R}(M)_{i \geqq 1}$ is nondecreasing for any finitely generated $R$-module $M$. We also show that the sequence $b_{i}(M)_{i \geqq 1}$ is strictly increasing for any finitely generated $R$-module $M$ if $\overline{(I+p) / p}$ is properly contained in $\overline{(J+p) / p}$ for any prime $p$ with depth $S_{p}=1$ (Theorem 1.8).

Rings of the form $S / I$ are not only generalized ones of the form $S / \underline{m} J$ but they have the following merit: To study nondecreasing of Betti numbers we may assume that $R$ is complete. Since $b_{i}^{R}(M)=b_{i}^{R}\left(M \otimes_{R} \hat{R}\right)$ where $\hat{R}$ is the $\underline{n}$-adic completion of $R$. Then by the Cohen structure theorem for complete local rings $R$ can be expressed as a homomorphic image of a complete regular ring $(S, \underline{m})$

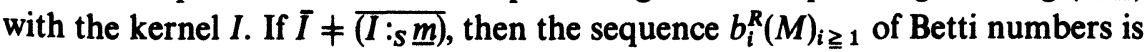
nondecreasing for any finitely generated $R$-module $M$ by Theorem 1.1. In section

Received November 24, 1988. 
2 we study the invariance of the module $\overline{(I: s \underline{m})} / \bar{I}$ under any regular presentations $S \rightarrow R \rightarrow 0$ of $R$, which is inspired from Theorem 1.1.

In section 3 we study 2-dimensional regular local rings and their contracted ideals. We prove that if an ideal $I$ of a 2 -dimensional regular. We prove that if an ideal $I$ of a 2-dimensional regular local ring $(S, \underline{m})$ is contracted, then $\bar{I} \neq \overline{(I: s \underline{m})}$ (Theorem 3.3). So if an artinian local ring $R$ of embedding dimesion 2 is a homomorphic image of a 2-dimensional regular local ring $S$ with the kernel $I$ contracted, then for any finitely generated $R$-module $M$ the sequence $b_{i}^{R}(M)_{i \geqq 1}$ of Betti numbers is nondecreasing by Theorem 1.1.

Finally, in section 4, we provide a positive answer to the problem of Avramov when $(R, \underline{n})$ is a artinian local ring of length at most 7 except possibly a complete intersection of length 6 or 7 . The problem is then true over a Cohen-Macaulay local ring of multiplicity at most 7 except possibly a complete intersection of multiplicity 6 or 7 by the change of Tor formula.

In the following sections we assume that every ring is commutative and noetherian.

ACKNOWLEDGMENT. I am grateful to my thesis advisor, Graig L. Huneke, for his guidance and many helpful discussions on this subject. Special thanks are also due to Luchezar L. Avramov and Jack Lescot for their advice and criticism. I am also indebted to David Rees for providing a crucial theorem.

\section{Betti numbers and the integral closure of ideals.}

Let $I$ be an ideal of a commutative noetherian ring $R$. An element $a$ of $R$ is said to be integral over $I$ if it satisfies an equation of the form

$$
a^{m}+\alpha_{1} a^{m-1}+\ldots+\alpha_{m}=0, \alpha_{i} \in I^{i} .
$$

The set of all elements of $R$ which are integral over $I$ is called the integral closure of $I$, and is denoted by $\bar{I}$. Note that $\bar{I}$ is an ideal of $R$ and $I=\bar{I}$. If $I=\bar{I}$, then $I$ is called complete.

For an $R$-module $M$ over a local ring $R \mu_{R}(M)$ shall denote the minimal number of generators of $M$ as an $R$-module.

ThEOREM 1.1. Let $(S, \underline{m})$ be a local ring and $R=S / I$ for an ideal $I$ of $S$. Let $J=(I: \underline{m})$. If $\bar{I} \neq \bar{J}$, then for any finitely generated $R$-module $M$

$$
b_{i+1}(M)-b_{i}(M) \geqq 0, i \geqq 1 .
$$

Proof. Consider a part of a minimal resolution of $M$,

$$
0 \rightarrow K \rightarrow R^{n} \stackrel{f}{\rightarrow} R^{q}
$$

where $K$ is the $(i+1)$ th syzygy of $M$, and $R^{n}\left(R^{q}\right)$ is the $i$ th module (the $(i-1)$ th module, respectively) in the minimal resolution of $M$. Then $b_{i+1}(M)=\mu_{R}(K)$, 
$b_{i}(M)=n, \quad K \subset \underline{m} R^{n}$ and $f\left(R^{n}\right) \subset \underline{m} R^{q}$. Since $J \underline{m} \subset I, \quad J R^{n} \subset K$. So $J R^{n} \subset K \subset \underline{m} R^{n}$. Let $\pi: S^{n} \rightarrow R^{n}$ be the natural projection, and let $A=\pi^{-1}(K)$. Then $\pi^{-1}\left(J R^{n}\right) \subset \pi^{-1}(K) \subset \pi^{-1}\left(\underline{m} R^{n}\right)$, that is, $J S^{n} \subset A \subset \underline{m} S^{n}$, Since $K=A / I S^{n}$ and $\underline{m} K=\left(\underline{m} A+I S^{n}\right) / I S^{n}, K / \underline{m} K \simeq A /\left(\underline{m} A+I S^{n}\right)$. So we can write $A=B+C$ for $S$-submodules $B$ and $C$ of $A$ such that $C \subset I S^{n}, \mu_{S}(C)=\operatorname{dim}_{S / m}\left(I S^{n}+\underline{m} A\right) / \underline{m} A$ and $\mu_{S}(A)=\mu_{S}(B)+\mu_{S}(C)$. Then $b_{i+1}(M)=\mu_{R}(K)=\operatorname{dim}_{S / \underline{m}} A / \underline{m} A-\operatorname{dim}_{S / \underline{m}}$ $\left(I S^{n}+\underline{m} A\right) / \underline{m} A=\mu_{S}(B)$.

We now choose an element $a$ of $J$ which is not integral over $I$, and put $N=S^{n} / B$. Since $I N=J N=A / B$, we have an element (by the determinant trick)

$$
x=a^{m}+\alpha_{1} a^{m-1}+\ldots+\alpha_{m}, \alpha_{i} \in I^{i},
$$

in $J$, and $x N=0$. If $x$ is nilpotent, then for some integer $t$,

$$
x^{t}=\left(a^{m}+\alpha_{1} a^{m-1}+\ldots+\alpha_{m}\right)^{t}=0,
$$

which is an integral equation for $a$ over $I$. This is a contraction. Therefore $x$ is a non-nilpotent element of $S$ which annihilates $N$. Hence $(x) S^{n} \subset B$. Let $T$ be the localization of $S$ at the multiplicative set $\left\{x^{i} \mid i \geqq 0\right\}$, and apply $\otimes_{S} T$ to $(x) S^{n} \subset$ $B \subset m S^{n}$. Then $T^{n} \subset B \otimes_{S} T \subset T^{n}$. Thus $B \otimes_{S} T=T^{n}$. Since the minimal number of generators of a module is not less than the minimal number of generators of a localization of the module,

$$
b_{i+1}(M)=\mu_{S}(B) \geqq \mu_{T}\left(B \otimes_{S} T\right)=n=b_{i}(M) .
$$

This finishes the proof of Theorem 1.1.

COROLlaRY 1.2. Let $(S, \underline{m})$ be a local and let I be an ideal of $S$ such that $R=S / I$ is of depth 0 . If $I$ is complete, then for any finitely generated $R$-module $M$ the sequence $b_{i}^{R}(M)_{i \geqq 1}$ of Betti is nondecreasing.

Proof. Let $J=(I: s \underline{m})$. Then $I \neq J$ since $R$ is of depth 0 . Therefore $I=$ $\bar{I} \neq \bar{J}$.

The order $o(I)$ of an ideal $I$ in a local ring $S$ is the largest integer such that $I \subset \underline{m}^{o(I)}$.

COROLLARY 1.3. Let $(S, \underline{m})$ be a regular local ring, and $R=S / I$ for an ideal I of $S$. Let $J=(I$ :s $\underline{m})$. If $o(J)<o(I)$, then for any finitely generated $R$-module $M$ the sequence $b_{i}^{R}(M)_{i \geqq 1}$ of Betti numbers is nondecreasing.

ProOF. Since any power $\underline{m}^{i}$ of the maximal ideal is integrally closed in a regular local ring, $\bar{I} \subset \underline{m}^{o(I)}$ but $J$ is not contained in $\underline{m}^{o(I)}$. Therefore $\bar{I} \neq \bar{J}$.

LEMMA 1.4. Let $(S, \underline{m})$ be a local ring and $J$ be an ideal of $S$. Then the following are equivalent:

(a) $J$ is nilpotent

(b) $\bar{J}=(\overline{0})$.

(c) $\bar{J}=\overline{J \underline{m}}$. 
Proof. Obviously, (a) if and only if (b), and (b) implies (c). So assume $\bar{J}=\bar{J} \underline{\text { }}$, and we will show that $J$ is nilpotent. Let $a$ be a generator of $J$ and $m$ be an integer such that

$$
a^{m}+\alpha_{1} a^{m-1}+\ldots+\alpha_{m}=0, \alpha_{i} \in(J \underline{m})^{i} .
$$

Hence $a^{m}=-\left(\alpha_{1} a^{m-1}+\ldots+\alpha_{m}\right) \in \underline{m} J^{m}$. Now we can choose a sufficiently large integer $t$ such that $J^{t}=\underline{m} J^{t}$. Therefore $J^{t}=0$ by Nakayama's Lemma.

We now reprove Proposition 2.1 [7].

COROLLARY 1.5. Let $(S, \underline{m})$ be a local ring and $J$ be a nonnilpotent ideal of $S$. Let $R=S / \underline{m} J$. Then for any finitely generated $R$-module $M$

$$
b_{i+1}(M)-b_{i}(M) \geqq 0, i \geqq 1 .
$$

Proof. If we let $I=J \underline{m}$ and $J_{1}=(I: s \underline{m})$, then $I=J \underline{m}=J_{1} \underline{m}$. $J_{1}$ is nonnilpotent since $J \subset J_{1}$. So by Lemma $1.4, \bar{I}=\overline{J_{1} \underline{m}} \neq \bar{J}_{1}$. The proof of the Corollary is complete by Theorem 1.1.

REMARK 1.6. Let $I$ be an irreducible $\underline{m}$-primary ideal of a local ring $(S, \underline{m})$, equivalently, $R=S / I$ is a 0 -dimensional Gorenstein ring. If $\mu(\underline{m} / I) \geqq 2$, then $\bar{I}=\overline{I: s \underline{m}}$. This is due to the example [5, section 3]: If $(R, \underline{n})$ is a 0 -dimensional Gorenstein local ring with embedding dimension at least 2 , then the Betti numbers $b_{i}^{R}\left(M_{k}\right)$ of $M_{k}=\operatorname{Hom}_{R}\left(s y z^{k}(R / \underline{n}), R\right)$ are strictly decreasing for $i=0, \ldots, k-1$.

Let $R$ be a local ring and let $M$ be a finitely generated $R$-module. We say that $M$ has $f$-rank $r$ if $M_{p}$ is a free $R_{p}$-module of constant rank $r$ for all associated primes $p$ of $R$. The f-rank of $M$ is denoted by frk $(M)$. For a closed subset $A$ of Spec $R$ we put

$$
\operatorname{codim} A:=\min \{\mathrm{ht} p \mid p \in A\}
$$

and let

$$
\mathrm{Nf}(M):=\left\{p \in \operatorname{Spec} R \mid M_{p} \text { is not a free } R_{p} \text {-module }\right\} .
$$

Bruns proved [4, Corollary 3] that if $M$ is a torsion free $R$-module with an f-rank, then

$$
\operatorname{codim} \mathrm{Nf}(M) \leqq \mu(M)+\mu\left(M^{*}\right)-2(\text { frk } M)+1
$$

where $M^{*}=\operatorname{Hom}_{R}(M, R)$.

Definition 1.7. Let $(S, \underline{m})$ be a local ring, $I$ an ideal of $S$ and let $J=(I: s \underline{m})$. We say that $I$ satisfies $\left(\mathrm{H}_{0}\right)$ if $\bar{I} \neq \bar{J}$ and that $I$ satisfies $\left(\mathrm{H}_{k}\right)$ for $k \geqq 1$ if for any prime $p$ of height $\leqq k$ and for any prime $p$ with depth $S_{p} \leqq 1, \overline{(I+p) / p} \neq \overline{(J+p) / p}$.

Note that $\left(\mathrm{H}_{k}\right)$ implies $\left(\mathrm{H}_{j}\right)$ for $j=0,1, \ldots, k$. 
THEOREM 1.8. Let $(S, \underline{m})$ be a local ring and let $I$ be an ideal of $S$. Let $J=(I: s \underline{m})$. If I satisfies $\left(H_{k}\right)$, then for any finitely generated non-free $R$-module $M$

$$
b_{i+1}(M)-b_{i}(M) \geqq k, i \geqq 1 .
$$

Proof. If $k=0$, then the case is done in Theorem 1.1. So assume that $k \geqq 1$. As in the proof of Theorem 1.1 , let $A=B+C$ and $N=S^{n} / B$ such that $b_{i+1}(M)=\mu(B), b_{i}(M)=n$ and $I N=J N=A / B$. Thus to prove that $b_{i+1}(M)-$ $b_{i}(M) \geqq k$, it is enough to show that $\mu(B)-n \geqq k$.

If we write $J=\left(a_{1}, \ldots, a_{t}, I\right)$, then associated with each $a_{i}$ we have

$$
x_{i}=a_{i}^{m}+\alpha_{i 1} a_{i}^{m-1}+\ldots+\alpha_{i m}, \alpha_{i j} \in I^{j},
$$

and $\left(x_{1}, \ldots, x_{t}\right) N=0$. Hence

$$
\left(x_{1}, \ldots, x_{t}\right) S^{n} \subset B \subset \underline{m} S^{n} .
$$

Suppose that ht $\left(x_{1}, \ldots, x_{t}\right) \leqq k$. Then $\left(x_{1}, \ldots, x_{t}\right) \subset p$ for some prime $p$ of height $\leqq k$. Hence $x_{i} \equiv 0$ modulo $p$ for all $i$ and this implies that $a_{i}+p \in \overline{(I+p) / p}$. So $\overline{(I+p) / p}=\overline{(J+p) / p}$, which contradicts $\left(\mathrm{H}_{k}\right)$. Therefore $\mathrm{ht}\left(x_{1}, \ldots, x_{t}\right) \geqq k+1$, and also grade $\left(x_{1}, \ldots, x_{t}\right) \geqq 2$ by the same argument. Since grade $\left(x_{1}, \ldots, x_{t}\right) \geqq 2$, (1) implies that $B$ is a torsion free $S$-module with f-rank $n$ and $B^{*} \simeq S^{n}$. So if we apply the result of Bruns, then

$$
\mu(B) \geqq \operatorname{codim} \mathrm{Nf}(B)-n+2 n-1 .
$$

Let $p$ be a prime of $S$ not containing $\left(x_{1}, \ldots, x_{t}\right)$ and localize the inclusion (1) at $p$. Then $S_{p}^{n} \subset B_{p} \subset S_{p}^{n}$. So $B_{p}=S_{p}^{n}$ and $p \notin \operatorname{Nf}(B)$. Hence codim $\mathrm{Nf}(B) \geqq \operatorname{ht}\left(x_{1}, \ldots, x_{t}\right)$. Substituting this in (2) yields

$$
\mu(B) \geqq h t\left(x_{1}, \ldots, x_{t}\right)+n-1 \geqq n+k,
$$

and the deseired conclusion of the Theorem follows from this.

Note that if the ideal $I$ satisfies $\left(\mathrm{H}_{1}\right)$, in other words, if $\overline{(I+p) / p}$ is properly contained in $\overline{(J+p) / p}$ for any prime $p$ with depths $S_{p}=1$, then the sequence $b_{i}^{R}(M)_{i \geqq 1}$ is strictly increasing for any finitely generated non-free $R$-module $M$.

Corollary 1.9. Let $(S, \underline{m})$ be a local ring, I be an ideal of $S$ of grade $\geqq 2$. Put $J=(I: s \underline{m})$. If $\bar{I}=\overline{J \underline{m}}$, then for any finitely generated non-free $R$-module $M$

$$
b_{i+1}(M)-b_{i}(M) \geqq h t I-1, i \geqq 1 .
$$

Proof. Obviously, $(J+p) / p$ is nonnilpotent for any prime $p$ of height $<$ ht $I$ and for any prime $p$ of grade $\leqq 1$. So by Lemma 1.4,

$$
\overline{(J \underline{m}+p) / p}=\overline{(I+p) / p} \neq \overline{(J+p) / p},
$$

that is, $I$ satisfies $\left(\mathrm{H}_{k}\right)$ for $k=\mathrm{ht} I-1$. Now the conclusion of the Corollary is immediate from 1.8 . 
Now we extend a Theorem of Ramras [12, Theorem 3.2A] to the non-domain case and give a better lower bound for $b_{i+1}(M)-b_{i}(M)$.

Corollary 1.10. Let $(S, \underline{m})$ be a local ring, $J$ be an ideal of grade $\geqq 2$ and let $R=S / \underline{m} J$. Then for any finitely generated non-free $R$-module $M$

$$
b_{i+1}(M)-b_{i}(M) \geqq \mathrm{ht} J-1, i \geqq 1 .
$$

PROof. If we let $I=J \underline{m}$ and $J_{1}=(I: s \underline{m})$, then $I=J \underline{m}=J_{1} \underline{m}$. Hence $\bar{I}=\overline{J_{1} \underline{m}}$. The proof of the Corollary is completely by Corollary 1.9.

EXAMPLES 1.11. Let $S=k[[x, y, z]]$ and $R=S / I$ for an ideal $I$ of $S$. If $I=\left(x^{2}, y^{3}, z^{5}, x y, y z, z x\right)$, then $J=(I$ :s $x, y, z)=\left(x, y^{2}, z^{4}\right)$, or if $I=\left(x^{2}, y^{3}, z^{4}, \mathrm{xy}\right.$, $\left.y^{2} z, z x\right)$, then $J=\left(x, y^{2}, z, y z^{3}\right)$. In both of these examples $x$ and $y^{2}$, simultaneously, cannot be integral over $I$ modulo any prime of height 1 . That is, such ideals $I$ satisfies $\left(\mathrm{H}_{1}\right)$. Therefore the sequence $b_{i}^{R}(M)_{i \geqq 1}$ is strictly increasing for any finitely generated non-free $R$-modulo $M$.

\section{The invariance of the module $\bar{J} / \bar{I}$.}

Let $(R, \underline{n})$ be a local ring and $M$ be a finitely generated $R$-module. To study nondecreasing of Betti numbers we may assume that $R$ is complete, since $b_{i}^{R}(M)=b_{i}^{R}\left(M \otimes_{R} \hat{R}\right)$ where the completion $\hat{R}$ of $R$ is flat over $R$. By the Cohen Structure theorem for complete local rings we then have a complete regular local $\operatorname{ring}(S, \underline{m})$ and an epimorphism $f: S \rightarrow R$. Considering Theorem 1.1 we may ask about the invariance of the module $\bar{J} / \bar{I}$ for any regular presentation $S \stackrel{f}{\rightarrow} R \rightarrow 0$, where $I=\operatorname{ker}(f)$ and $J=(I$ :s $\underline{m})$.

From now on let's denote $C_{R}(S, f)$ for $\bar{J} / J$ when $(S, \underline{m})$ and $(R, \underline{n})$ are local rings with an epimorphism $f: S \rightarrow R, I=\operatorname{ker}(f)$ and $J=(I: s \underline{m})$. In this section, we study the following problems, when $R$ is complete.

(1) Are the $C_{R}(S, f)$ isomorphic for all complete regular local rings $S$ and for all epimorphisms $f: S \rightarrow R$ ?

(2) If $C_{R}(S, f) \neq 0$ for some complete local ring $S$ with an epimorphism $f: S \rightarrow R$, does it follow that $C_{R}(T, g) \neq 0$ for any complete regular local ring $T$ and for any epimorphism $g: T \rightarrow R$ ?

LEMMA 2.1. Let $\left(S_{i}, \underline{m}_{i}\right), i=1,2$ and $(R, \underline{n})$ be local rings with epimorphisms $f: S_{2} \rightarrow S_{1}$ and $g: S_{1} \rightarrow R$. If $C_{R}\left(S_{1}, g\right) \neq 0$, then $C_{R}\left(S_{2}, g \circ f\right) \neq 0$.

Proof. Let $I_{1}=\operatorname{ker}(g), I_{2}=\operatorname{ker}(g \circ f)$ and $J_{i}=\left(I_{i}: \underline{m}_{i}\right)$, for $i=1,2$. Consider the homomorphism $\bar{f}$,

$$
\bar{f}: J_{2}+\bar{I}_{2} / \bar{I}_{2} \rightarrow J_{1}+\bar{I}_{1} / I_{1}
$$

which is induced by $f$. $f$ is well-defined and onto since $f\left(\bar{I}_{2}\right) \subset \bar{I}_{1}$ and $f\left(J_{2}\right)=J_{1}$. 
Therefore $J_{1}+\bar{I}_{1} / \bar{I}_{1} \neq 0$ implies $J_{2}+\bar{I}_{2} / \bar{I}_{2} \neq 0$. Hence $C_{R}\left(S_{2}, g \circ f\right) \neq 0$ if $C_{R}\left(S_{1}, g\right) \neq 0$.

The proof of the following theorem is in a letter from D. Rees. I wish to express my gratitude to $D$. Rees for showing me this crucial result.

THEOREM 2.2. (D. Rees) Let $(S, \underline{m})$ be a regular local ring and I be a complete ideal which contains an element $x \in \underline{m}-\underline{m}^{2}$. Then $I /(x) R$ is complete.

LEMMA 2.3. Let $\left(S_{i}, \underline{m}_{i}\right), i=1,2$ be regular local rings and $(R, \underline{n})$ be a local ring. Suppose we have epimorphisms $f: S_{2} \rightarrow S_{1}$ and $g: S_{1} \rightarrow R$. Then $C_{R}\left(S_{1}, g\right) \simeq$ $C_{R}\left(S_{2}, g \circ f\right)$.

ProOF. It is enough to prove the lemma when $\operatorname{dim} S_{2}=\operatorname{dim} S_{1}+1$. Let $I_{1}=\operatorname{ker}(g), I_{2}=\operatorname{ker}(g \circ f), J_{i}=\left(I_{i}: s_{i} \underline{m}_{i}\right)$ for $i=1,2$, and $\operatorname{ker}(f)=(x)$ for $x \in \underline{m}-\underline{m}^{2}$. Then $x \in I_{2}$. Consider the map $\bar{f}$,

$$
\overline{f:} \bar{J}_{2} \rightarrow \bar{J}_{1} / \bar{I}_{1}
$$

which is induced by $f . \bar{f}$ is well-defined since $f\left(\bar{J}_{2}\right) \subset \bar{J}_{1}$. Note that

$$
J_{1}=f\left(J_{2}\right) \subset f\left(\bar{J}_{2}\right) \subset \bar{J}_{1} .
$$

By Theorem $2.2 f\left(\bar{J}_{2}\right)$ is complete, so $f\left(\bar{J}_{2}\right)=\bar{J}_{1}$ and $\bar{f}$ is onto. Similarly $f\left(\bar{I}_{2}\right)=\bar{I}_{1}$. Hence $\operatorname{ker}(\bar{f})=\bar{I}_{2}+(x)=\bar{I}_{2}$. Therefore $C_{R}\left(S_{1}, g\right) \simeq C_{R}\left(S_{2}, g \circ f\right)$.

LEMMA 2.4. Let $(R, \underline{n})$ be a complete local ring containing a field $k \simeq R / \underline{n}$. Suppose we have $k$-epimorphisms $f_{i}: S_{i} \rightarrow R$ where $\left(S_{i}, \underline{m}_{i}\right)$ are complete regular local rings containing $k$ with $\operatorname{dim} S_{i}=\operatorname{edim}(R), i=1,2$. Then there exists a $k$-isomorphism g: $S_{1} \rightarrow S_{2}$ such that $f_{1}=f_{2} \circ g$.

Proof. Let $\underline{m}_{1}=\left(X_{1}, \ldots, X_{d}\right), d=\operatorname{edim}(R)$ and $x_{i}=f_{1}\left(X_{i}\right)$, then $\underline{n}=$ $\left(x_{1}, \ldots, x_{d}\right)$. Let $Y_{i}$ be a lifting of $x_{i}$ under $f_{2}$. We claim that $\underline{m}_{2}=\left(Y_{1}, \ldots, Y_{d}\right)$. Since $\operatorname{edim}(R)=\operatorname{dim} S_{2}, \operatorname{ker}\left(f_{2}\right) \subset \underline{m}_{2}^{2}$. Hence

$$
\left(Y_{1}, \ldots, Y_{d}\right)+\operatorname{ker}\left(f_{2}\right)=\underline{m}_{2},
$$

which implies that $\left(Y_{1}, \ldots, Y_{d}\right)=\underline{m}_{2}$ by Nakayama's Lemma. Now the Cohen structure theorem for complete regular local rings gives the identification $S_{1}=$ $k\left[\left[X_{1}, \ldots, X_{d}\right]\right]$ and $S_{2}=k\left[\left[Y_{1}, \ldots, Y_{d}\right]\right]$. Define a $k$-homomorphism $g: S_{1} \rightarrow S_{2}$ by $g\left(X_{i}\right)=Y_{i}$. Then $g$ is a $k$-isomorphism such that $f_{1}=f_{2} \circ g$. Then

THEOREM 2.5. Let $(R, \underline{n})$ be a complete local ring containing a field $k \simeq R / \underline{n}$.

(1) The $C_{R}(S, f)$ are isomorphic for all complete regular local rings $(S, \underline{m})$ containing $k$ and for all k-epimorphisms $f: S \rightarrow R$.

(2) If $C_{R}(S, f) \neq 0$ for some complete local ring $S$ containing $k$ with a $k$-epimor- 
phism $f: S \rightarrow R$, then $C_{R}(T, g) \neq 0$ for any complete regular local ring $T$ containing $k$ and for any $k$-epimorphism $g: T \rightarrow R$.

ProOF. (1) Immediate from Lemma 2.3 and 2.4.

(2) By the Cohen structure theorem for complete local rings, there is a complete regular local ring $T$ containing $k$ with a $k$-epimorphism $g: T \rightarrow S$ [11, Theorem (31.1)]. Then $C_{R}(T, g \circ f) \neq 0$ by Lemma 2.1. Now (2) is complete by (1).

We conclude this section with a question whose positive answer suffices the invariance of $C_{R}(S, f)$ for any complete regular local ring $S$ and for any epimorphism $f: S \rightarrow R$.

QUESTION 2.6. Let $(R, \underline{n})$ be a complete local ring and let $S_{1}=S_{2}$ be a formal power series ring over $R / \underline{n}$ or over a $v$-ring of $R / \underline{n}$ with $\operatorname{dim} S_{1}=\operatorname{edim}(R)$ or $\operatorname{edim}(R)+1$. Suppose there exist epimorphisms $f_{i}: S_{i} \rightarrow R$, for $i=1,2$. Is there an isomorphism $g: S_{1} \rightarrow S_{2}$ such that $f_{1}=f_{2} \circ g$ ?

\section{Contracted ideals of 2-dimensional regular local rings.}

Let $(S, \underline{m})$ be a 2-dimensional regular local ring and $x \in \underline{m}-\underline{m}^{2}$. An ideal $I$ of $S$ is called contracted from $S[\underline{m} / x]$ if $I=I S[\underline{m} / x] \cap S$. An ideal $I$ is contracted from $S[\underline{m} / x]$ if and only if $(I: s x)=(I: s \underline{m})$ [8, Proposition 2.1]. For any $\underline{m}$-primary ideal $I$ of $S \mu(I) \leqq o(I)+1$ by Hilbert-Burch Theorem, and an $\underline{m}$-primary ideal $I$ of $S$ is contracted if and only if $\mu(I)=o(I)+1[8$, Proposition 2.3].

LEMMA 3.1. Let I be an m-primary ideal of a 2-dimensional regular local ring $(S, \underline{m})$ and let $J=(I: s \underline{m})$. Then

(1) $\mu(J)-\mu(I)+1=\operatorname{dim} I / \underline{m} J$.

(2) If $\mu(J)-\mu(I)=-1$ or 0 , that is, if $\operatorname{dim} I / \underline{m} J=0$ or 1 , then I satisfies $\left(H_{0}\right)$. That is, $\bar{I} \neq \bar{J}$.

Proof. (1) From the resolution of $S / \underline{m}$ it follows that $\operatorname{Tor}_{2}^{S}(S / I, S / m)$ is isomorphic to $J / I$ and by applying the Hilbert-Burch theorem for the resolution of $S / I$ we can see that $\operatorname{Tor}_{2}(S / I, S / \underline{m}) \simeq J / I$, is a $S / \underline{m}$-vector space of dimension $\mu(I)-1$. Hence

$$
\operatorname{dim} I / \underline{m} J=\operatorname{dim} J / \underline{m} J-\operatorname{dim} J / I=\mu(J)-(\mu(I)-1) .
$$

(2) If $\mu(J)-\mu(I)=-1$, then $I=\underline{m} J$. So $I \neq J$. Otherwise $J$ is nilpotent by Lemma 1.4. This is a contradiction since $S$ is 2-dimensional. If $\mu(J)-\mu(I)=0$, then $\operatorname{dim} I / \underline{m} J=1$. So $I=(f, \underline{m} J)$ for some $f \in I-\underline{m} J$. Suppose $J$ is integral over $I$, then $J /(f)$ is integral over $\underline{m} J+(f) /(f)$ in $S /(f)$. This is a contradiction since $\operatorname{dim} S /(f)=1$. Therefore $J$ cannot be integral over $I$. 
We remark a useful fact implied in the proof of Lemma 3.1(2).

REMARK 3.2. Let $(S, \underline{m})$ be a noetherian local ring, $I$ an $\underline{m}$-primary ideal of $S$ and let $J=(I: s \underline{m})$. If $I \subset\left(\underline{m} J, I_{1}\right)$ and $\operatorname{dim} S / I_{1} \geqq 1$ for some ideal $I_{1}$ of $S$, then $\bar{I} \neq \bar{J}$.

THEOREM 3.3. Let I be an m-primary ideal of a 2-dimensional regular local ring $(S, \underline{m})$ and let $J=(I: s \underline{m})$. If $I$ is contracted, then $\bar{I} \neq \bar{J}$.

PROof. If $I$ is contracted, then so is $J$. Since for some $x \in \underline{m}-\underline{m}^{2}$

$$
(I: \underline{m}): x=I: \underline{m} x=(I: x): \underline{m}=(I: \underline{m}): \underline{m},
$$

we have $\mu(I)=o(I)+1$ and $\mu(J)=o(J)+1$. However $o(J)=o(I)$ or $o(J)=$ $o(I)-1$ since $\underline{m} J \subset I \subset J$. Therefore

$$
\mu(J)-\mu(I)=o(J)-o(I)=-1,0 .
$$

Now the Theorem is complete by Lemma 2.1 .

REMARK 3.4. Suppose $(R, \underline{n})$ is an artinian local ring of embedding dimension 2 , then it is a homomorphic image of a 2-dimensional regular local ring $(S, \underline{m})$ with the kernel $I \subset m^{2}$, since artinian local rings are complete. So if $I$ is contracted, equivalently $o(I)+1=\mu(I)$, then $I$ satisfies $\left(H_{0}\right)$ and the sequence $b_{i}^{R}(M)_{i \geqq 1}$ of Betti numbers of any finitely generated $R$-module $M$ is nondecreasing by Theorem 1.1.

\section{Lower multiplicity Cohen-Macaulay local rings and Betti numbers.}

In this section we study the nondecreasing of the sequence of Betti numbers over lower multiplicity Cohen-Macaulay local rings. First we discuss the nondecreasing of the sequence of Betti numbers over hypersurfaces.

REMARK 4.1. Let $(R, \underline{n})$ be an artinian local ring of embedding dimension 1 . Then it is a homomorphic image of a $\operatorname{DVR}(S, x)$. So $R \simeq S /\left(x^{h+1}\right)$ for some integer $h$ and $J=\left(x^{h+1} ;_{S} x\right)=\left(x^{h}\right)$ is not integral over the defining ideal $I=\left(x^{h+1}\right)$. Therefore for any finitely generated $R$-module $M$ the sequence $b_{i}(M)_{i \geqq 1}$ of Betti numbers is nondecreasing. Hence over a $d$-dimensional hypersurface $R$ the sequence $b_{i}^{R}(M)_{i \geq d+1}$ of Betti numbers is nondecreasing for any finitely generated $R$-module $M$ by the change of Tor formula (see Theorem 4.3).

For an artinian local ring $(R, \underline{n})$ and a finitely generated $R$-module $M$, let $l(M)$ denote the length of $M$ and $h(R)$ be the largest integer such that $\underline{n}^{h(R)} \neq 0$. The Hilbert function $H_{R}(t)$ of $R$ is the polynomial, $1+e_{1} t+\ldots+e_{h(R)} t^{h(R)}$, where $e_{i}=\operatorname{dim}_{R / \underline{n}} \underline{n}^{i} / \underline{n}^{i+1}$.

It is a result of Lescot that if $\underline{n}^{3}=0$, then for any finitely generated $R$-module 
$M$ the sequence $b_{i}^{R}(M)$ is eventually nondecreasing [9, Theorem B, Proposition 3.9].

We now quote a result of Gasharov and Peeva.

LEMMA 4.2. Let $(R, \underline{n})$ be an artinian local ring. If

$$
\delta_{1}(R):=2 \operatorname{edim}(R)+h(R)-l(R)-1 \geqq 1,
$$

then for any finitely generated $R$-module $M$ the sequence $b_{i}^{R}(M)$ is eventually nondecreasing.

ProOF. [6, Proposition 2.2]

THEOREM 4.3. Let $(R, \underline{n})$ be an artinian local ring of length at most 7 and let $M$ be a finitely generated $R$-module. Then the sequence $b_{i}^{R}(M)$ of Betti numbers is eventually nondecreasing except possibly $R$ is a complete intersection of length 6 or 7.

PROOF. By Remark 4.1 we may assume that $e_{1} \geqq 2$ and also by a result of Lescot we may assume that $h(R) \geqq 3$. If $e_{1} \geqq 3$, then $\delta_{1}(R) \geqq 6+3-7-1=1$. So the assertion is true by Lemma 4.2. Hence it is enough to prove the Theorem for $e_{1}=2$ and $h \geqq 3$. Then $l(R) \geqq 1+2+1+1=5$. If $l(R)=5$, then $H_{R}(t)=$ $1+2 t+t^{2}+t^{3}$ and $\delta_{1}(R)=1$. Hence the Theorem is true. So we may assume that $R$ is not a complete intersection of length 6 or 7 .

Now express $R$ as a homomorphic image of a 2-dimensional regular local ring $(S, \underline{m})$ with the kernel $I \subset \underline{m}^{2}$ and let $J=(I: s \underline{m})$. If $o(I) \geqq 4$, then $H_{R}(t)=$ $1+2 t+3 t^{2}+4 t^{3}+\ldots$. Hence $l(R) \geqq 10$ and this is not a case of the Theorem. If $o(I)=3$, then $H_{R}(t)=1+2 t+3 t^{2}+t^{3}$ (note, $e_{3} \neq 0$ since $h(R) \geqq 3$ ). In this case $R$ cannot be Gorenstein, otherwise $R$ is self-injective, so we have

$$
l\left(0:_{R} \underline{n}^{2}\right)=l\left(R / \underline{n}^{2}\right)=7-4 \geqq l\left(\underline{n}^{2}\right)=4,
$$

a contradiction. Since $R$ is not Gorenstein, $J \notin \underline{m}^{3}$ but $I \subset \underline{m}^{3}$, so $o(J)=2$ and $o(I)=3$. Thus the Theorem is true by Corollary 1.3. If $o(I)=2$, then $\mu(I) \leqq 3$ by the Hilbert-Burch Theorem. Since we have assumed $R$ is not complete intersection, $\mu(I)=3$. Therefore $I$ is contracted and the Theorem is now complete by Theorem 3.3.

COROllary 4.4. Let $(S, \underline{m})$ be a Cohen-Macaulay local ring of multiplicity at most 7 and let $M$ be a finitely generated $S$-module. Then the sequence $b_{i}^{S}(M)$ of Betti numbers is eventually nondecreasing unless $S$ is a complete intersection of multiplicity 6 or 7 .

Proof. There exists $S$-regular sequence $x_{1}, \ldots, x_{d}(d=\operatorname{dim} S)$, such that the multiplicity of $S$ is equal to the length of $R\left(=S /\left(x_{1}, \ldots, x_{d}\right)\right)$. Since the $S$-regular 
sequence $x_{1}, \ldots, x_{d}$ is also regular on $\operatorname{syz}^{d}(M)$, we can apply the change of Tor formula,

$$
\operatorname{Tor}_{i+d}^{S}(M, S / \underline{m}) \simeq \operatorname{Tor}_{i}^{S}\left(\operatorname{syz}^{d}(M), S / \underline{m}\right) \simeq \operatorname{Tor}_{i}^{R}\left(\operatorname{syz}^{d}(M) \otimes_{S} R, S / \underline{m}\right) .
$$

This implies that

$$
b_{i+d}^{S}(M)=b_{i}^{S}\left(\operatorname{syz}^{d}(M)\right)=b_{i}^{R}\left(\operatorname{syz}^{d}(M) \otimes_{S} R\right), i \geqq 1 .
$$

The proof is now complete by applying Theorem 4.3 for $R$.

RemarK 4.5. It is a result of Avramov that over a complete intersection $R$ the sequence $b_{i}^{R}(M)$ of Betti numbers of any finitely generated $R$-module $M$ has strong polynomial growth [2, Proposition 4.3]. That is, there exist polynomials $p(i)$ and $q(i)$ of the same degree and with the same leading term such that

$$
p(i) \leqq b_{i}^{R}(M) \leqq q(i), i \gg 0 .
$$

Over a complete intersection the degree of the polynomials is one less than the complexity of the module $M$. If the degree of the polynomial is 0 , then the sequence of Betti numbers is eventually constant and any minimal resolution of $M$ is periodic with period 2. Otherwise, both even and odd Betti numbers are strictly increasing. Therefore, over a Cohen-Macaulay local ring $R$ of multiplicity at most 7, we have provided a positive answer to a problem of Ramras [13]: There are only two possibilities, either the sequence $b_{i}^{R}(M)$ is eventually constant, or $\lim _{i} b_{i}^{R}(M)=\infty$.

\section{REFERENCES}

1. L. Avramov, Local algebra and rational homotopie Algebrique et Algebre Locale, Asterisque 113-114 (1984), 15-43.

2. L. Avramov, Module of finite virtual projective dimension, Reports, Univ. of Stockholm, No 31 (1987).

3. L. Avramov, Homological asymptotes of modules over local rings, to appear in the proceedings of Micro Program in Commutative Algebra MSRI Berkeley (1987), Springer-Verlag, Lecture Notes in Math.

4. W. Bruns, The Eisenbud-Evans generalized principal ideal theorem and the determinantal ideals, Proc. Amer. Math. Soc. 83 (1981), 19-24.

5. D. Eisenbud, Homological algebra on a complete intersection with an application to group representations, Trans. Amer. Math. Soc. 260 (1980), 35-64

6. V. Gasharov and I. Peeva, Boundedness versus Periodicity over commutative local rings, preprint (1987).

7. E. H. Gover and M. Ramras, Increasing sequences of Betti numbers, Pacific J. Math. 87 (1980), 65-68.

8. C. Huneke, Complete ideals in two-dimensional regular local rings, to appear in the proceedings of Micro Program in Commutative Algebra MSRI Berkeley (1987), Springer-Verlag, Lecture Notes in Math.

9. J. Lescot, Asymptotic properties of Betti numbers of modules over certain rings, J. Pure Appl. Algebra 38 (1985), 287-298. 
10. J. Lescot, Séries de Poicaré modules inertes, preprint (1987).

11. M. Nagata, Local Rings, Robert E. Krieger Pub. Company, 1975.

12. M. Ramras, Betti numbers and the reflexive modules, in $\mathbf{R}$. Gordon ed., Ring Theory, Academic Press (1972), 297-308.

13. M. Ramras, Sequences of Betti numbers, J. Algebra 66 (1980), 193-204.

14. D. Rees, a letter (1988).

15. O. Zariski and P. Samuel, Commutative algebra Vol II, Van Nostrand, Amsterdam, 1960.

DEPARTEMENT OF MATHEMATICS

PURDUE UNIVERSITY

WEST LAFAYETTE. INDIANA 47907

U.S.A.
CURRENT ADRESS:

DEPARTMENT OF MATHEMATICS

MICHIGAN STATE UNIVERSITY

EAST LANSING, MI 48824-1027

U.S.A. 